\title{
Health Care Professionals and Intersex Conditions
}

Joel Frader, MD, MA; Priscilla Alderson, PhD; Adrienne Asch, PhD; Cassandra Aspinall, MSW, ACSW; Dena Davis, JD, PhD; Alice Dreger, PhD; James Edwards, PhD; Ellen K. Feder, PhD;

Arthur Frank, PhD; Lisa Abelow Hedley, JD; Eva Kittay, PhD; Jeffrey Marsh, MD;

Paul Steven Miller, LLB; Wendy Mouradian, MD; Hilde Nelson, PhD; Erik Parens, PhD

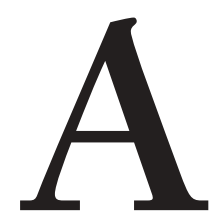

s part of a larger project, a multidisciplinary group convened by The Hastings Center, Garrison, NY, met to consider medical, psychosocial, and ethical issues associated with the care of children born with atypical genitalia or later found to have other conditions now commonly grouped together as "intersex." These children may have congenital adrenal hyperplasia, gonadal dysgenesis, hypospadias, partial or complete androgen insensitivity syndrome, etc. This commentary reflects the deliberations of the group, which concluded that none of the appearance-altering surgeries need to be performed quickly; families with children with intersex conditions require multidisciplinary care; children with intersex conditions deserve to know the truth about their bodies; families and health care professionals will benefit from rigorous longitudinal studies; and health care professionals need additional training about intersex conditions and sexual health generally.

Parents and physicians of children with intersex conditions rarely know about the condition before birth. The recognition that a child does not appear typically "male" or "female" prompts distress, confusion, and uncertainty, no matter the age at diagnosis. When health care professionals attempt to determine a "true" biological or underlying sex of the child or inappropriately communicate an urgent need to undertake a surgical "fix," they wrongly substitute these interventions for focus-
From the Department of General Academic Pediatrics, Children's Memorial Hospital, Chicago, Ill (Dr Frader); the Feinberg School of Medicine, Northwestern University, Evanston, Ill (Dr Frader); the Social Science Research Unit, Institute of Education, University of London, London, England (Dr Alderson); the Department of Women's Studies, Wellesley College, Boston, Mass (Dr Asch); the Craniofacial Center, Children's Hospital and Regional Medical Center, Seattle, Wash (Ms Aspinall); the Cleveland-Marshall College of Law, Cleveland, Ohio (Dr Davis); the Lyman Briggs School, College of Natural Science (Dr Dreger) and the Department of Philosophy (Dr Nelson), Michigan State University, East Lansing; the Department of Philosophy, Furman University, Greenville, SC (Dr Edwards); the Department of Philosophy and Religion, American University, Washington, DC (Dr Feder); the Department of Sociology, University of Calgary, Calgary, Alberta (Dr Frank); the Children of Difference Foundation, New York, NY (Ms Hedley); the Department of Philosophy, State University of New York, Stony Brook University, Stony Brook (Dr Kittay); Washington University, St Louis, Mo (Dr Marsh); Kids Plastic Surgery, St Louis (Dr Marsh); US Equal Employment Opportunity Commission, Washington, DC (Mr Miller); Pediatric Dentistry and Health Services, University of Washington, Seattle (Dr Mouradian); and The Hastings Center, Garrison, NY (Dr Parens). ing on the families' discomfort, guilt, and/or sense of shame. All should understand that a decision to raise an infant as a boy or girl in no way depends on surgery, per se. Chromosome analysis and pelvic ultrasonography, as well as some endocrine testing, may help make a precise diagnosis; however, test results do not accurately predict later sexual identification.

\section{See also page 500}

The need for slow, deliberate, and careful explanations; psychological and social support; offers of contact with other people who have experienced similar situations (especially adults who have lived with the child's condition); and exploration of a wide range of alternative courses of action seem essential. In the past, intervention has sometimes begun long before disclosure to or consultation with the child, despite a lack of physiologic justification. Initial surgeries may beget more operations in order to correct problems arising from those performed previously. Drug treatments may further alter the child's appearance and/or mood, again 
without the individual's consent or sufficient rationale. Absent compelling physiologic reasons to operate, decisions must rest on psychosocial considerations. Available data do not provide adequate reasons for using surgery in most cases before the child has the developmental capacity to participate in decision making. Future research must address this question.

Many sent down the road of early "treatment" now express their discontent. ${ }^{1}$ Physical discomfort associated with repeated surgical procedures and/or the sequelae of those interventions have dominated their lives. A good number have diminished sexual responsiveness and pleasure as a result of "cosmetic" surgeries undertaken when they had no opportunity to participate in decision making. Such reports and the results of retrospective reviews have led 2 groups of pediatric urologists to urge reconsideration of early surgery. ${ }^{2-4}$ Some patients have experienced humiliation at the hands of physicians (violations of privacy and dignity) and endured secrecy and confusion in and disruption of their relationships, especially with parents-who have themselves suffered in the web of deception-and health care professionals. ${ }^{5}$ Because of all this, treatment approaches recently recommended by the American Academy of Pediatrics, ${ }^{6}$ Elk Grove Village, Ill, and a task force of the Lawson Wilkins Pediatric Endocrine Society, Stanford, Calif, and the European Society for Paediatric Endocrinology, ${ }^{7}$ London, England, have begun to receive reappraisal by other medical professionals. ${ }^{8-10}$ Notably, the guidance documents lack justification based on valid clinical investigation. While critiques of the recommendations by experts may signal growing variability in practice, many presumably continue to rely on these professional recommendations. Our own experience suggests substantial disagreement within the endocrinology and surgical communities in pediatrics, even within single institutions.

Controversy remains about the levels of satisfaction with surgical outcomes and general well-being among those treated for intersex conditions in the past. We have no rigorous and unbiased studies of the long-term effects of medical interventions, in part because of practices of keeping individuals with intersex conditions in the dark about their histories. Without diagnostic candor, meaningful follow-up studies are difficult, if not impossible. Ethical practice requires such studies. To their credit, some who treat patients with intersex conditions, such as pediatric urologist Ian Aaronson, MD, have begun efforts to design and conduct proper studies, as discussed at an October 2001 meeting at the National Institute of Child Health and Human Development. ${ }^{8}$ However, nothing has come to fruition thus far. When research planning does begin, it should address: (1) how the patients have felt and now feel about their medical care; (2) what should count as positive or negative outcome measures (subject satisfaction with sexual functioning, subject comfort with gender identity, genital appearance, and so on); (3) the impact of changing surgical techniques-results of operations done 15 to 20 years ago may not predict outcomes for newer surgeries; and (4) deficiencies in some completed follow-up studies ${ }^{11,12}$ conducted by members of the original treatment teams that raise questions about the willingness of patients to ex- press candidly negative feelings to those who provided well-intentioned care. Secrecy breeds shame and does not serve the interest of patients and other family members, though it has shielded some adults-parents and medical professionals-from accountability for their decisions. While studies will have to confront the heterogeneous nature of the intersex population, we believe sufficient commonalities exist to permit meaningful research results.

We conclude:

- The field should undertake a comprehensive assessment of actual practice. What do endocrinologists, pediatric surgeons, pediatric urologists, and others recommend? What operations do surgeons use, on which patients, at what ages, and with what outcomes?

- Surgical techniques that make children's genitals appear more normal and operations that produce less physical damage than earlier techniques would not alone provide justification for surgery. Good clinical practice demands attention to the social and cultural context into which children are born. Health care professionals should acknowledge that surgery will not end discrimination against persons with physical differences from the "norm."

- None of the appearance-altering surgeries need be done urgently. Surgery to normalize appearance without the consent of the patient lacks ethical justification, in most cases. Some surgeons argue that technical considerations warrant intervention in the first year of life to fashion typical female anatomy. However, we do not know if surgical expediency outweighs the psychosocial and ethical considerations of waiting until the patient can participate in decision making. The irrevocable nature of surgery should make everyone extremely cautious.

- Families with children with an intersex condition require a comprehensive package of services immediately following diagnosis. The approach should include access to suitably qualified social workers and/or other mental health professionals and referral to parentto-parent programs, support groups, and adults living with the same or a similar condition. Centers lacking social work/mental health personnel with specific expertise in intersex conditions should nevertheless provide team care using psychosocially trained professionals interested in and experienced with helping families face medical and social ambiguity across extended periods. Teams offering care for patients with intersex conditions should add requisite experts as available or assist current staff with appropriate professional development. The lack of local social work and/or mental health expertise cannot become an excuse for circumventing needed psychosocial support.

- Children have the right to know about their bodies. Professionals and parents should tell children, in an age-appropriate fashion, about how and why they have anatomical differences from others. The differences should provide opportunities to explore the value of individuality and diversity, not occasions for humiliation and shaming.

- Ethical practice demands rigorous follow-up studies. The goal of achieving real patient benefit mandates 
comprehensive research with patients who have intersex conditions. The principal focus should be how patients assess their own well-being, including peer relationships through their childhood, adolescence, and adulthood. In retrospective studies, every effort should be made to include surgically treated individuals and those who have not had surgery. Treatment centers should cooperate in multicentered, prospective studies designed to provide better epidemiological data on the incidence and prevalence of intersex conditions and compare the results of alternative courses of action (eg, no infant surgery vs surgery) chosen by families after careful counseling and full, deliberative informed consent about the risks, benefits, and unknowns of the possible paths. Both retrospective and prospective studies require systematic, reliable methods and must contain qualitative elements to permit subjects to tell their stories and express their feelings freely. Study designs must candidly address researcher bias. Adequate studies may require independence from treating endocrinologists and urologists.

- Health care professionals need considerably more education about intersex conditions, including recognition, precise diagnosis, the development of gender identity, and sexual health. This should occur in schools of medicine, nursing, social work, clinical psychology, and so on, in addition to postgraduate clinical training programs. As part of these efforts, educators should address how the helping professions can address inappropriate and unhealthy attitudes toward individuals, such as those with intersex conditions, who appear different and/or behave differently from most others and whose abilities differ from the norm. Medicine can reflect common social prejudices or it can help society develop tolerance and appreciation for human diversity. We endorse the latter.
Accepted for publication January 5, 2004.

This study was supported by grant RZ-20715 from the National Endowment for the Humanities, Washington, DC. Corresponding author: Joel Frader, MD, MA, General Academic Pediatrics, Children's Memorial Hospital, 2300 Children's Plaza-16, Chicago, IL 60614 (e-mail: j-frader@northwestern.edu).

\section{REFERENCES}

1. Dreger A, ed. Intersex in the Age of Ethics. Hagerstown, Md: University Publishing Group; 1999.

2. Alizai NK, Thomas FM, Lilford RJ, et al. Feminizing genitoplasty for congenital adrenal hyperplasia: what happens at puberty. J Urol. 1999;161:1588-1591.

3. Creighton SM, Minto CL, Steele SI. Objective cosmetic and anatomical outcomes at adolescence of feminizing surgery for ambiguous genitalia done in childhood. Lancet. 2001;358:124-125.

4. Minto CL, Liao LM, Woodhouse CRJ, Ransley PG, Creighton SM. The effect of clitoral surgery on sexual outcome in individuals who have intersex conditions with ambiguous genitalia: a cross-sectional study. Lancet. 2003;361:1252-1257.

5. Kemp BD. Sex, lies and androgen insensitivity syndrome. CMAJ. 1996;154:1829.

6. American Academy of Pediatrics Committee on Genetics Section on Endocrinology and Section on Urology. Evaluation of the newborn with developmental anomalies of the external genitalia. Pediatrics. 2000;106:138-142.

7. Joint LWPES/ESPE CAH Working Group. Consensus statement on 21hydroxylase deficiency. J Clin Endocrinol Metab. 2002;87:4048-4053.

8. Blizzard RM. Intersex issues: a series of continuing conundrums. Pediatrics. 2002 110:616-621.

9. Daaboul J, Frader J. Ethics and the management of the patient with intersex: a middle way. J Pediatr Endocrinol Metab. 2001;14:1575-1583.

10. Creighton S, Ransley P, Duffy P, et al. Regarding the consensus statement on 21-hydroxylase deficiency from the Lawson Wilkins Pediatric Endocrine Society and the European Society for Paediatric Endocrinology. J Clin Endocrinol Metab. 2003;88:3455

11. Migeon CJ, Wisniewski AB, Gerheart JP, et al. Ambiguous genitalia with perineal scrotal hypospadias in 46,XY individuals: long-term medical, surgical, and psychosexual outcome. Pediatrics [serial online]. 2002;110:e31. Available at: http: //pediatrics.org/cgi/content/full/110/3/e31. Accessed January 28, 2004.

12. Migeon CJ, Wisniewski AB, Brown TR, et al. 46,XY intersex individuals: phenotype and etiologic classification, knowledge of condition, and satisfaction with knowledge in adulthood. Pediatrics [serial online]. 2002;110:e32. Available at: http://pediatrics.org/cgi/content/full/110/3/e32. Accessed January 28, 2004.

\section{Invited Critique}

Reality vs Recommendations in the Care of Infants With Intersex Conditions

A 15-member multidisciplinary panel from the Hastings Center Surgically Shaping Children Group, Garrison, NY, has proposed guidelines for the management of children with intersex conditions. While based on a comprehensive and careful deliberation of multiple issues pertaining to intersexuality, these guidelines fail to acknowledge many of the obstacles and uncertainties faced by practicing health care professionals today. Thus, the goal of this commentary is to offer an appraisal of these recommendations under the lens of a pediatric endocrinologist grappling with the real life challenges of providing compassionate and responsible care to infants with intersex conditions and their families. Each conclusion made by the Hastings Center will be presented and discussed.

According to the Hastings Center group, none of the appearance-altering surgeries need be done urgently and surgery to normalize appearance without the consent of the patient lacks ethical justification, in most cases.

Decisions regarding whether and when to perform early genital surgery in infants with intersex disorders are undeniably among the most difficult. ${ }^{1}$ Historical claims that formation of a stable gender identity requires concordant genitalia in terms of appearance have legitimately been questioned and rejected by many. However, the opposite view that genital surgery be uniformly deferred until the patient is able to give consent cannot be supported by available information. The greatest concern revolves around the potential psychosocial sequelae of growing up with genitalia discrepant from those of siblings and other children. No data currently exist that shed light on to what extent this concern is warranted, if at all. An additional flaw in the blanket recommendation to withhold all nonessential early genital surgery is that it overlooks the fundamental role of parents in ensuring a child's physical and emotional well-being. Above and beyond factors such as prenatal androgen exposure, appearance of the genitalia, and surgery or no surgery, the most important determinant of outcome may be an individual family's ability to accept and unconditionally love their child. ${ }^{2}$ Recent reports of comparable degrees of satisfaction and dissatisfaction with sex of rearing, body image, and sexual function in 46, XY undervirilized males raised either male or female would seem to support this view. ${ }^{3}$ If parents maintain a strong prejudice in favor of surgery despite a

(continued) 
complete education about its potential risks, the effects of unilaterally denying this option for a decade or more could surely be disastrous. Therefore, until rigorous comparisons of psychosexual function in patients receiving early genital surgery using modern techniques vs no early genital surgery have been conducted, only 2 conclusions may safely be made. First, the ultimate decision regarding early surgery rests with the parents and should be made in the context of their own cultural beliefs only after a detailed and comprehensive review of all options, opinions, and available outcome data. ${ }^{4}$ Affording parents this authority does not present an ethical dilemma, since in our society all major decisions regarding minor children are traditionally made by parents. Second, when early genital surgery is performed, it should be undertaken by surgeons in centers of excellence with particular expertise in the care of patients with intersex conditions. ${ }^{5}$

According to the Hastings Center group, families with children with intersex conditions require multidisciplinary care and the lack of local social work and/or mental health expertise cannot become an excuse for circumventing needed psychosocial support.

It has long been acknowledged that psychological counseling and support should be an integral component of the multidisciplinary standard of care for children with intersex disorders, and we agree with this approach. Unhappily, it has also been demonstrated that few, if any, centers actually follow such a model. ${ }^{6}$ In reality, it often falls to the pediatric endocrinologist to guide the family through the initial distress and subsequent decision-making process that follow the birth of an infant with an intersex condition, a situation that is clearly suboptimal. However, failure to provide mental health expertise may not indicate deliberate circumvention but may result from a deep reluctance on the part of the family to comply with psychological followup. Having personally been told on more than one occasion that "God is my psychiatrist," physicians can no more force families to avail themselves of counseling than request that they reorient their religious beliefs. Efforts to train qualified mental health practitioners must continue with the goal of establishing in situ intersex teams through which it is conveyed to families that a multidisciplinary approach is assumed in both initial and ongoing management.

According to the Hastings Center group, children with intersex conditions deserve to know the truth about their bodies.

Previous medical practices advocating secrecy and the withholding of accurate information from patients with intersex conditions have been rightfully denounced. ${ }^{7}$ In their place we are left with a vague mandate to provide age-appropriate explanations to children with intersex conditions regarding why and how they are different from others. Precisely who should deliver this information, with what words, at what time, and in what form (written vs verbal vs pictorial) has never been explicitly stated. Thus, we muddle along in our attempts to provide full disclosure in ways that respect the psychological vulnerability of the child, the inherent sensitivity of the subject, and the parents' wishes regarding whether to reveal the diagnosis, which are sometimes in direct conflict with our own. Clearly, health care professionals taking care of children with intersex conditions need more specific guidance than that which is currently offered.

According to the Hastings Center group, ethical practice demands rigorous follow-up studies.

Agreed! It is essential to recognize that anecdotal case reports, no matter how compelling, should never dictate medical practice. ${ }^{8}$ Rather, prospective, long-term multicenter trials are vital in establishing outcomes and informing clinical management. Advances in molecular genetic analysis will enhance the ability to make a precise diagnosis, while the development of creative consortia focused on intersexuality will foster scientific inquiry and collaborative research endeavors.

According to the Hastings Center group, health care professionals need additional training about intersex conditions and sexual health generally.

While information regarding human sexuality and the need for tolerance regarding diversity should be incorporated in the education of all health care professionals, it is unlikely that a general inclusion of these topics will contribute substantially to the care of children with intersex conditions. Rather, the development and implementation of intensive short-term programs geared toward the infusion of individuals with special expertise into the healthcare system would likely be a more effective approach. Participants in such programs could include physicians, nurses, social workers, psychologists, parents, and adult patients who could provide an important liaison role between individual families and the health care team.

Much has been written recently regarding the management of children with intersex conditions. Despite apparent differences, significant common ground may be identified. The next phase in the shifting evolution of current management protocols must be devoted to obtaining objective information regarding outcomes in various conditions. During this critical time, it will be essential to avoid polarization and to engage in an honest, empathetic exchange with the common goal of providing the best possible care to children with intersex conditions and their families.

Erica A. Eugster, MD Indianapolis, Ind

I would like to thank my colleagues Jack Fuqua, MD; Ora Pescovitz, MD; Kathy Bethin, BA; and Linda DiMeglio, MD, for helpful suggestions and thoughtful review of this commentary.

Corresponding author and reprints: Erica A. Eugster, MD, Pediatric Endocrinology, Riley Hospital, Room 5960, 702 Barnhill Dr, Indianapolis, IN 46202 (e-mail: eeugster@iupui.edu).

1. Blizzard RM. Intersex issues: a series of continuing conundrums. Pediatrics. 2002;110:616-621.

2. Berenbaum SA. Management of children with intersex conditions: psychological and methodological perspectives. Growth Genetics Horm. 2003;19:1-7.

3. Migeon CJ, Wisniewski AB, Gearhart JP, et al. Ambiguous genitalia with perineoscrotal hypospadius in 46,XY individuals: long-term medical, surgical, and psychosexual outcome. Pediatrics. 2002;110:1-10.

4. Daaboul J, Frader J. Ethics and the management of the patient with intersex: a middle way. J Pediatr Endocrinol Metab. 2001;14:1575-1583.

5. Joint LWPES/ESPE CAH Working Group. Consensus statement on 21-hydroxylase deficiency from the Lawson Wilkins Pediatric Endocrine Society and the European Society for Paediatric Endocrinology. J Clin Endocrinol Metab. 2002;87:4048-4053.

6. Chase C. What is the agenda of the intersex patient advocacy movement? Endocrinologist. 2003;13:240-242.

7. Groveman SA. Sex, lies and androgen insensitivity syndrome. CMAJ. 1996;154:1829-1833.

8. Zucker KJ. Evaluation of sex- and gender-assignment decisions in patients with physical intersex conditions: a methodological and statistical note. J Sex Marital Ther. 2002;28:269-274. 